\title{
Embedded Systems Design for Low Energy Consumption
}

\author{
Michael A. Schuette, Ph.D., Motorola, Inc. \\ John R. Barr, Ph.D., Motorola, Inc.
}

\begin{abstract}
This tutorial covers the circuit fundamentals of CMOS circuits which contribute to the consumption of energy in portable products, as well as guidelines for the design of systems in order to reduce energy consumption and prolong battery life. Circuit fundamentals will include a definition of terms, basic circuit elements, laws of operation, and basic circuit theory applying energy consumption. We will then present three major principles of energy reduction: reducing number of transitions, reducing the amount of switched capacitance, and reducing the operating voltage. Several guidelines that can be applied during the system design process which utilize the three major principles.
\end{abstract}

\section{Introduction}

Embedded system containing portable devices

Typically, consists of several portable devices each providing its user with local computational capability. The devices are capable of communicating with each other through RF links to an intermediate communication network or directly through point-to-point RF or IR links.

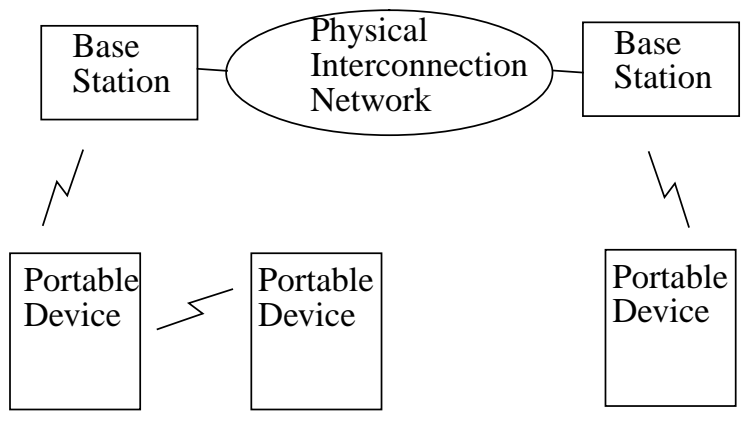

Design objectives

1. Reduce energy consumption of portable devices
2. Reduce avg. power consumption of stationary devices

1 and 2 may conflict since, given two systems where the first consumes less energy but in a shorter period of time the first may have a higher average power consumption

This tutorial will focus on design objective 1

\section{MOS digital circuit fundamentals}

Definition of terms

$$
\begin{array}{ll}
\mathrm{R}=\text { resistance } & \mathrm{C}=\text { capacitance } \\
\mathrm{I}=\text { current } & \mathrm{V}=\text { voltage } t \\
\mathrm{P}=\text { power }=\mathrm{I}^{*} \mathrm{~V} & \mathrm{E}=\text { energy }=\int_{0}^{t} \mathrm{P} d t
\end{array}
$$

NMOS transistor operation

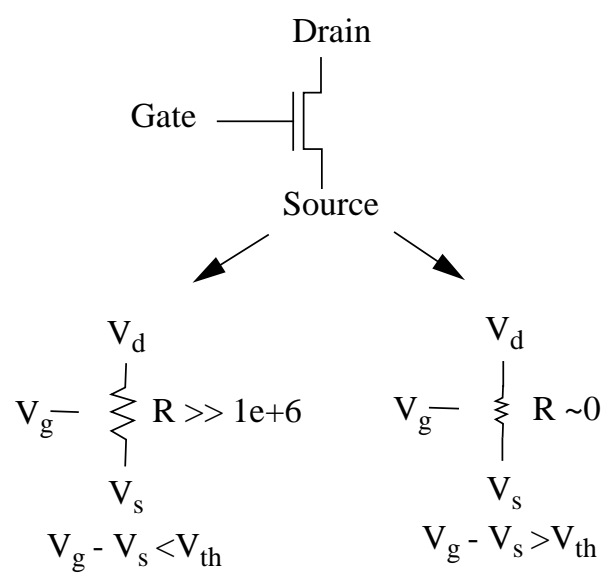

(a)

(b)

Figure 1.

The NMOS transistor acts as a switch. When the gate-source voltage is less than a voltage, $V_{\text {th }}$, the transistor has a high resistance between its drain and source, as in Fig. 1(a). When the gate-source voltage is greater than $\mathrm{V}_{\mathrm{th}}$ the transistor has a low resistance between its drain and source, as in Fig. 1(b). 
Sources of energy consumption

The CMOS inverter, as shown at the top of Fig. 2, will be used to illustrate the primary sources of energy consumption in a typical CMOS logic gate

1. Capacitive load current
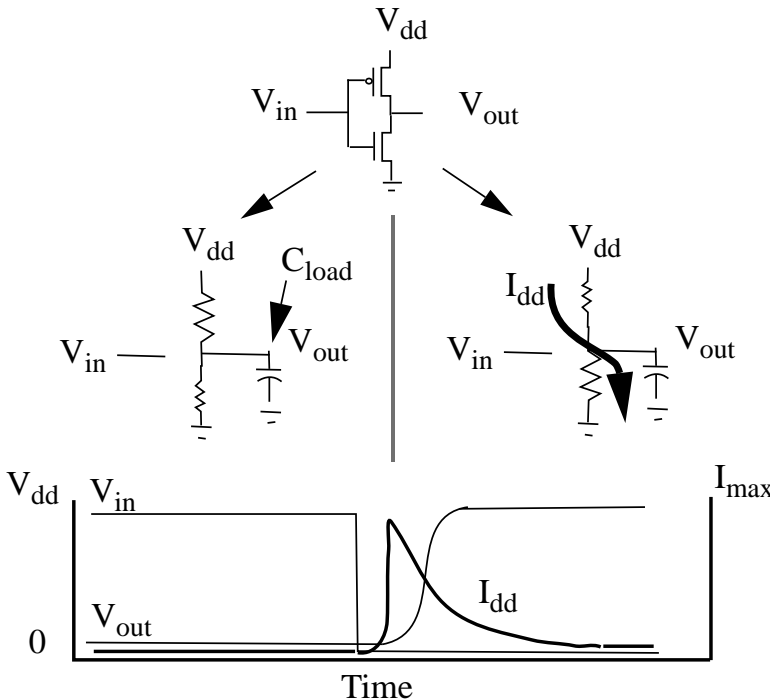

Figure 2.

Occurs when the output voltage of the inverter changes from a low voltage to a high voltage

When the output is low, resistance from the output to $\mathrm{V}_{\mathrm{dd}}$ is high and resistance to ground is low, causing the load capacitance to be discharged

As the resistance from the output to $\mathrm{V}_{\mathrm{dd}}$ goes low and resistance to ground goes high, due to a change in the input voltage, the output voltage goes high and the output capacitance is charged

$\mathrm{E}=0.5 \cdot \mathrm{C} \cdot \mathrm{V}_{\mathrm{dd}}^{2}$ per transition

Primary source of energy consumption in CMOS

\section{Short circuit current}

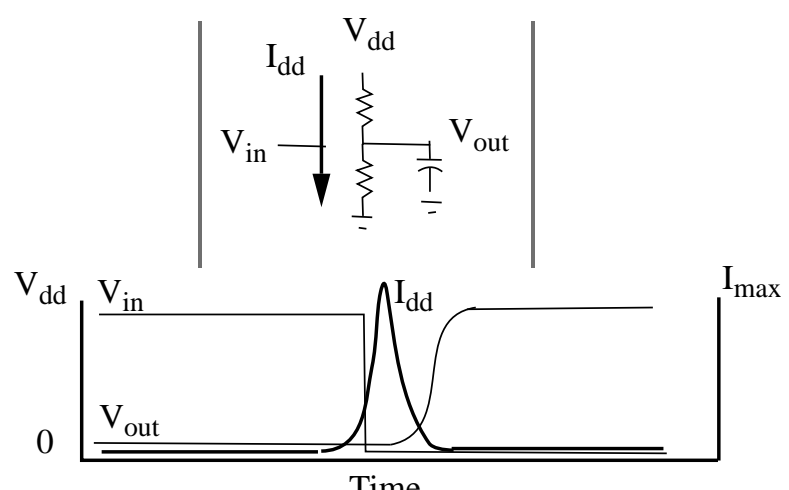

Figure 3.

Occurs when output voltage is between high and low values

Resistance to $\mathrm{V}_{\mathrm{dd}}$ and ground is moderate setting up a conductive path between them

$\mathrm{E}=\left(\left(\mathrm{V}_{\mathrm{dd}}-\left(\mathrm{V}_{\mathrm{tn}} \cdot \mathrm{V}_{\mathrm{tp}}\right) \cdot \mathrm{I}_{\max } \cdot\left(\mathrm{T}_{\text {rise }} \cdot \mathrm{T}_{\text {fall }}\right)\right) / 2\right)$ per transition

Typically $20 \%$ of total energy consumption

3. Static current
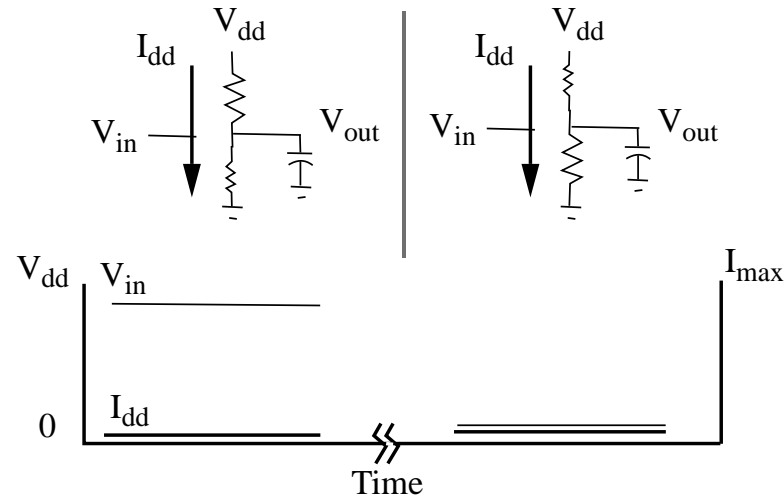

Figure 4.

Occurs when output voltage is high or low

Finite resistance between $V_{d d}$ and ground results in a small, but non-zero current between them

$\mathrm{E}=\int_{0}^{t} \frac{\mathrm{V}_{\mathrm{dd}}^{2}}{\mathrm{R}_{1} \cdot \mathrm{R}_{\mathrm{d}}} \cdot d t$

Usually significant only in devices inactive for large periods of time 


\section{Principles of energy consumption reduction}

To a first approximation $\mathrm{E}=0.5 \cdot \mathrm{C} \cdot \mathrm{V}_{\mathrm{dd}}^{2} \cdot \mathrm{N}$, where $\mathrm{N}=$ \# transitions

Three principles:

\section{Reduce operating voltage}

\section{Reduce capacitance being switched}

\section{Reduce number of transitions}

Important: It's the number of transitions that counts and not the frequency with which they occur

To achieve significant reductions in each of these three factors

a) The goal of low energy must be part of the design process

b) Low energy design must be approached at all levels of design, including the system, algorithm and device architecture levels

\section{Design process}

\section{Guideline 1: Make energy a design constraint}

1. Maintain an energy budget - set energy targets for parts of the design from the start

\section{Assign responsibility for meeting energy goals}

3. Track progress through use of power estimation tools

Spreadsheets - advantages and disadvantages

+ Easy to generate early in design cycle with minimal design information

- Can be difficult to update as design changes

- Prone to error as spreadsheet model not linked directly to design tools and database

- Prone to error as assumptions made concerning component activity not linked to profiling information
Architectural Power Analysis Tools [1] advantages and disadvantages

+ Solves many of the drawbacks of spreadsheets

- Not quite soup yet, no commercially available tools

4. Review progress through regularly scheduled design reviews

\section{System level}

Network communication often a significant source of portable device energy consumption

\section{Guideline 2: Use compression}

Reduces number of "transitions" on the network

Many compression techniques exist - designer must select one(s) which match network communication characteristics and application

Important network and application characteristics:

Real time vs. non-real time - in real time systems compression/decompression typically must be simpler so that they can be performed in less time

Ability to tolerate loss of data - systems that can tolerate loss of data can use lossy compression schemes which achieve higher compression rates

Packetized vs. non-packetized - in packetized networks, if compression is to be done on a per packet basis, packet size will influence the relative effectiveness of compression schemes

\section{Guideline 3: Use unbalanced communication}

Shift energy use to parts of system where it is not a concern

Example: image compression [2]

Consider a system where the image originates and is compressed in a portion of the system where 
energy consumption is not a concern, such as the network, and then transmitted to and decompressed on a portable device

Computational requirements of two methods for image compression are shown below

\begin{tabular}{|c|c|c|}
\hline & \multicolumn{2}{|c|}{ Computation Reqs/pixel } \\
\hline Method & compression & decompression \\
\hline $\begin{array}{l}\text { Transform } \\
\text { (DCT) } \\
\text { (row-col. fast } \\
\text { algorithm) }\end{array}$ & $\begin{array}{l}4 \text { multiply } \\
8 \text { additions } \\
6 \text { mem. access }\end{array}$ & $\begin{array}{l}4 \text { multiply } \\
8 \text { additions } \\
6 \text { mem. access }\end{array}$ \\
\hline $\begin{array}{l}\text { Vector Quant. } \\
\text { (Tree search - } \\
\text { differential CB) }\end{array}$ & $\begin{array}{l}8 \text { multiply } \\
8 \text { additions } \\
8 \text { mem. access }\end{array}$ & 1 mem. access \\
\hline
\end{tabular}

Vector quantization requires only one memory operation per pixel to decompress vs. four multiplies, eight additions and six memory operations per pixel for DCT. However, compression is more energy consumptive for vector quantization than for DCT. Thus, vector quantization saves energy over DCT in the portable device at a cost in energy in the network

\section{Algorithm level}

Surprisingly, many guidelines at algorithm and device architecture level are similar to ones for achieving high performance

\section{Guideline 4: Exploit parallelism}

Use parallelism to maintain throughput while reducing clock rate and operating voltage [3]

Example: execution path parallelism

Consider the execution path of a typical processor as shown in Fig. 5 (sans GP register bank)

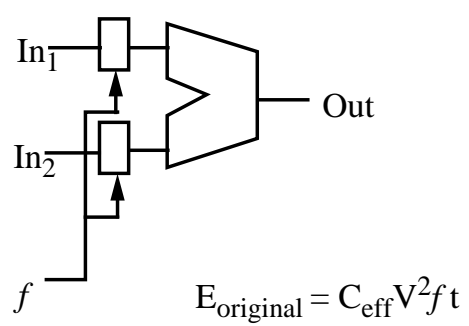

Figure 5.

The input latches are clocked with frequency $f$ so that $\mathrm{f}$ operations/sec are performed

Now consider an execution path with two ALU's, each of which is clocked at half the rate of the original, as in Fig. 6

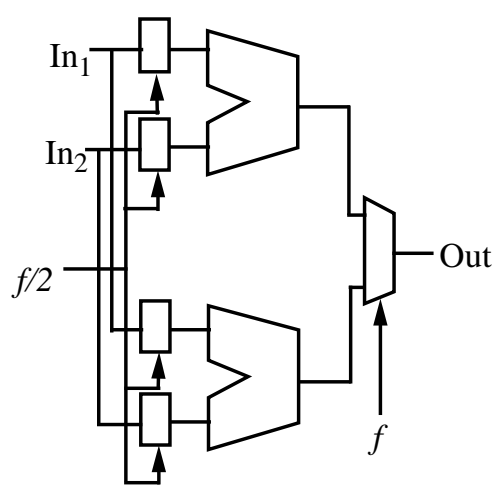

$\mathrm{E}_{\text {parallel }}=2 \mathrm{C}_{\text {eff }}(\mathrm{V} / 2)^{2}(f / 2) \mathrm{t}=\underline{.25 \mathrm{E}_{\text {original }}}$

Figure 6.

Reduction in clock speed, due to parallelism, allows operating voltage to be reduced by an approximately proportional amount

Reduction in operating voltage reduces energy consumption proportional to $\mathrm{V}^{2}$, and in this example by a factor of 4

Energy consumption reduction depends on ability of the software to exploit the parallelism in the hardware. Greater parallelism in algorithm allows for greater utilization of parallelism in hardware

Significant gains possible - research shows a reduction of up to 7 times demonstrated for simple circuits

Guideline 5: Choose an energy efficient algorithm 
Energy to perform a given function depends upon the algorithm chosen to implement the function

Example: cascade vs. parallel realization of a discrete time system [4]

Consider the discrete time system, whose transfer function, $\mathrm{H}(\mathrm{z})$, is:

$\mathrm{H}(\mathrm{z})=\frac{3 z^{2}+3.6 z+0.6}{z^{2}+0.1 z-0.2}$

The flow diagram of the "cascade" realization of this system is given in Fig. 7. It requires 4 multiplies, 4 additions and 5 memory accesses to implement

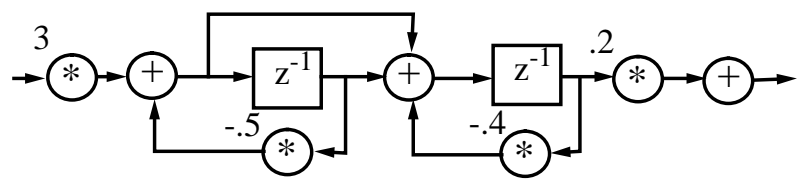

$\mathrm{N}$

* multiply by $\mathrm{N}$

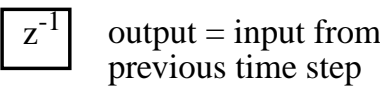

( ) sum all inputs

Figure 7.

The flow diagram of the "parallel" realization of this system is given in Fig. 8. It requires 5 multiplies, 4 additions and 5 memory accesses to implement

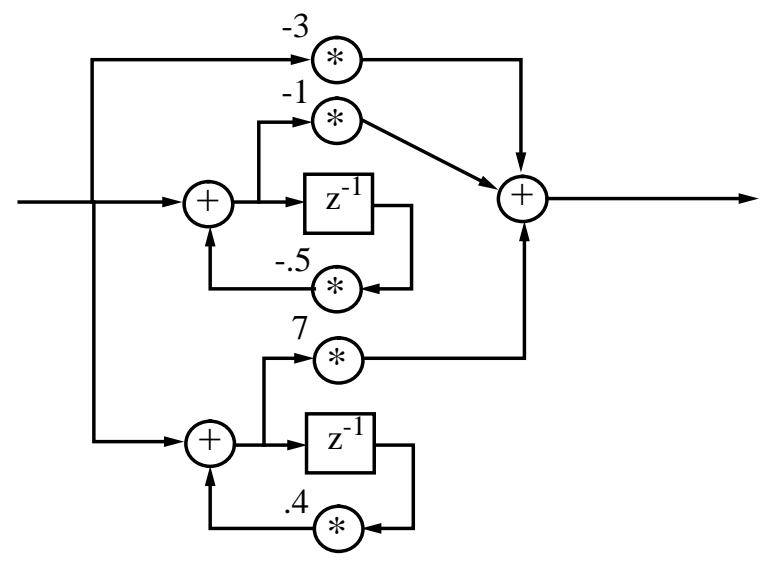

Figure 8 .

The "cascade" form in this case uses $20 \%$ fewer multiplies
Potentially large difference for some algorithms

Efficiency vs. parallelism - more parallel algorithm may require more operations but use less energy if it allows system to run at a lower operating voltage

\section{Guideline 6: Apply optimizing transformations}

Improve energy efficiency of an algorithm by reordering operations, substituting for energy consumptive operations, changing where variables are stored, etc.

Example: product of a complex variable by a complex constant

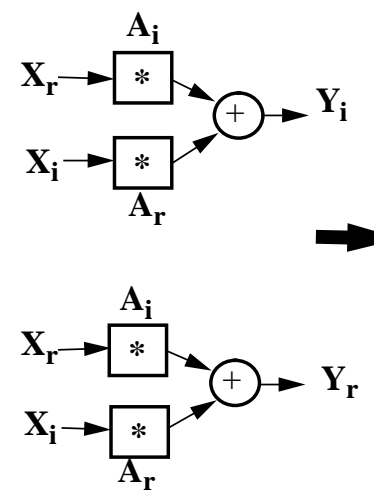

(a)

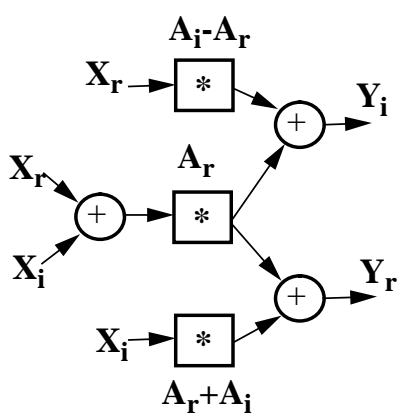

(b)
Figure 9.

In Fig. 9(a), the real and imaginary terms are generated independently

In Fig. 9(b), the real and imaginary terms of the constant are combined and used to eliminate one multiplication at the expense of one addition

Since additions tend to consume less energy than multiplies, this transformation saves energy

Similar to compiler optimizations for performance

Efficiency vs. parallelism - same trade-off as for Guideline 5, since (a) is more parallel than (b) but uses less energy consumptive operations

\section{Device architecture}

Guideline 6: Match hardware to algorithm 
Processor - Choose processor with instruction set, data path width, functional units suitable to algorithm

16-bit RISC reduces memory traffic by $\sim 25 \%$ over 32-bit RISC, with comparable performance, for limited memory systems [5]

Least energy consumptive DSP processor varies according to the application [6], as shown in the table below

Energy per Function (in W)

\begin{tabular}{|l|l|l|l|}
\hline Function & $\begin{array}{c}\text { ADI } \\
\text { ADSP } \\
2103\end{array}$ & $\begin{array}{c}\text { Motorola } \\
\text { DSP56L002 }\end{array}$ & $\begin{array}{c}\text { TI } \\
\text { TMS320LC } \\
51\end{array}$ \\
\hline \hline Real FIR & 6.32 & 7.86 & $\mathbf{4 . 8 6}$ \\
\hline IIR & .35 & .36 & .51 \\
\hline Vect. Max & .88 & $\mathbf{. 7 4}$ & .95 \\
\hline 256 Pt FFT & 89.08 & $\mathbf{7 0 . 2 2}$ & 113.83 \\
\hline
\end{tabular}

Support logic - Implement often used functions in hardware

Often functions are less energy consumptive when performed on special-purpose hardware than when performed in software on general-purpose hardware

Thus, the addition of hardware timers, specialpurpose coprocessors/functional units tend to reduce energy consumption

\section{Guideline 7: Localize communication}

Map functions in algorithm to hardware such that interchip communication is minimized

$$
\begin{aligned}
& \text { May result in a trade-off vs. maximal } \\
& \text { exploitation of parallelism inherent in } \\
& \text { algorithm }
\end{aligned}
$$

Use memory hierarchy [7]

Use distributed vs. centralized resources [2]

Example: motion compensation

Uniprocessor
Single high speed ALU

4ns memory

Supply voltage 5V

$\mathrm{f}_{\text {clock }}=250 \mathrm{Mhz}$

$\mathrm{E}=88.2 \mathrm{e}-6 \mathrm{~J}=0.5 \cdot \mathrm{C}_{\text {uni }} \cdot 5^{2} \cdot 2.5 \mathrm{e}+8 \cdot 1.26 \mathrm{e}-5$

Multiprocessor

48 concurrent processors

Supply voltage $=1.1 \mathrm{~V}$

$\mathrm{f}_{\text {clock }}=1 \mathrm{Mhz}$

$\mathrm{E}=60 \mathrm{e}-9 \mathrm{~J}=0.5 \cdot \mathrm{C}_{\text {multi }} \cdot 1.1^{2} \cdot 1 \mathrm{e}+6 \cdot 1.2 \mathrm{e}-5$

$\mathrm{C}_{\text {multi }}=.27 \cdot \mathrm{C}_{\text {uni }}$

Some reduction in the effective capacitance due to operations being performed on smaller processors, but remaining reduction due to lack of communication over highly capacitive global busses

\section{Guideline 8: Reduce resource sharing}

Resource sharing tends to decrease signal correlation and hence increase switching activity [2]

Example: dual counters

Two counters in Fig. 10(a) are multiplexed onto a bus in alternate cycles. Successive values for each counter are highly correlated, i.e. they differ in only two bit positions on average. However, successive values appearing on shared bus differ significantly depending upon skew between counts.

Non-shared configuration of Fig. 10(b) keeps the output data streams of the two counters from interfering with each other. 


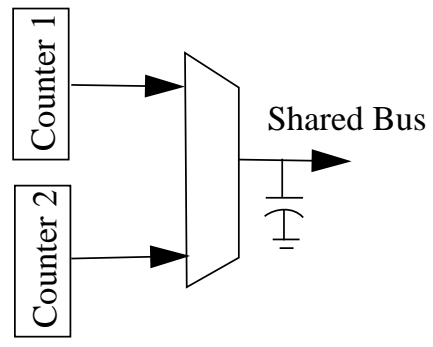

(a)

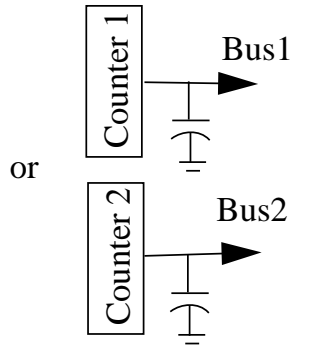

(b)
Results assume counters alternate use of shared bus every cycle, with random skew between counts

Figure 10.

Other examples include: multiplexed data busses within an execution unit and multiplexed external address and data busses occuring on some processors Does not always increase switching activity depends on autocorrelation of each data stream vs. correlation between data streams when sharing resources

\section{Guideline 9: Partition design}

Partition design into subcircuits whose power level can be independently controlled

Power down subcircuits when not in use

This allows the system to keep its energy consumption closer to the minimum level necessary to perform the function at hand

Higher resolution partitions tend to yield greater energy savings but savings are eventually offset by energy needed to operate circuitry controlling power level of sub-circuits

\section{Conclusion}

Energy consumption is proportional to $\mathrm{C} \cdot \mathrm{V}^{2} \cdot \mathrm{N}$

Energy consumption reduction must be a part of the design process and addressed at all levels of design

Careful choice of algorithm, hardware, and mapping of algorithm to hardware can yield orders of magnitude reduction

J. Rabaey, R. Brodersen, et al. at UCB have designed and built a portable multimedia terminal with total power consumption of $50 \mathrm{~mW}$, excluding power of display - order of magnitude less than comparable designs

Once system design in complete, need to continue to address energy consumption at the logic and physical design level

\section{References}

[1] Landman, P. and J.M. Rabaey, "Black-Box Capacitance Models for Architectural Power Analysis", Int'l Workshop on Low Power Design, April, 1994, pp 165-170.

[2] Rabaey, J., "Tutorial 3: Low Power System Design: Solutions and Challenges", Design Automation Conference, June, 1994.

[3] Chandrakasan, A., S. Sheng, and R. Brodersen, "Low-Power CMOS Digital Design", IEEE Journal of Solid-State Circuits, Vol. 27, No. 4, April, 1992, pp. 473-483.

[4] Stanley, W., G. Dougherty, and R. Dougherty, "Digital Signal Processing", Reston Publishing, 2nd Edition, 1984

[5] Bunda, J., D. Fussell, R. Jenevein, and W.C. Athas, "16-Bit vs. 32-Bit Instructions for Pipelined Microprocessors", 20th Int'l. Symposium on Computer Architecture, May 16-19, 1993, pp. 237246

[6] Buyer's Guide to DSP Processors, Berkeley Design Technologies Inc., 1994

[7] Wuytack, S., F. Catthoor, F. Franssen, L. Nachtergaele, and H. DeMan, "Global communication and memory optimizing transformations for low power systems", Int'l Workshop on Low Power Design, April, 1994, pp 203-208. 